\title{
Research on Knowledge Representation in Expert System
}

\author{
Xianjiu Guo ${ }^{a}$, Yue Li ${ }^{\text {b }}$ \\ Dalian Ocean University, Dalian, China; \\ a879753691@qq.com, b33977300@qq.com
}

Keywords: Knowledge Representation, Expert System, Semantic Network

\begin{abstract}
This paper is based on expert system, and described the expert system knowledge representation method, compared production rules and predicate logic notation, which led to the advantages of production rules. In the semantic network representation, general procedures of semantic representation were proposed according to semantic relations, and example was given out for verification.
\end{abstract}

\section{Introduction}

Knowledge Representation connect knowledge factor to the knowledge, to facilitate people to identify and understand knowledge. Knowledge representation is the premise and basis of knowledge organization, and any knowledge organization methods are to be established on the basis of the knowledge representation.

The knowledge in expert system refers to the concept, events and processes organized by computer and their structures can be denoted fully. Therefore, the knowledge in the expert system has its specific scope and structure, and also can be encoded. The knowledge representation in expert system is generally divided into the following three categories. The first category is the concept which is extracted from the real world. The second kind includes events, behavior and state abstracted from the real world. In addition, the third class is about the process, namely, it not only includes the description of their behavior, but also includes the explanation of the changing conditions and other relationships[1-2].

In expert systems, more widely used is the expert system based on rules. Its main characteristic is the application of the production representation method, which is also called rule representation. This representation was first proposed by BOST in 1943, and in 1972 used in artificial intelligence research by Newell and Simon. In addition, widely applied representations include predicate logic representation and semantic network representation [3].

\section{Production Rules}

Comparison between Predicate Logic and Production Rules. Production is mainly used to represent causal concern, said there are two main forms:

IF P THEN Q, or: $\mathrm{P} \rightarrow \mathrm{Q}$

Where $\mathrm{P}$ as a prerequisite for a causal relationship, and $\mathrm{Q}$ as a result.

The generally form of predicate logic $\mathrm{Q}\left(x_{1}, x_{2}, \ldots x_{n}\right)$ can be used to represent certain types of knowledge, in which $\mathrm{Q}$ is the predicate name, $x_{1}, x_{2}, \ldots x_{n}$ are individuals. For example, Student ( $\mathrm{Li}$ Ming) represents the fact that Li Ming is a student. If $x_{1}, x_{2}, \ldots x_{n}$ are single individual constants, then they are called first-order predicate, which is different from higher-order logic, and it does not allow the quantification of nature. For example, first-order logic can represent an object is red, but it is not suitable for expression of the nature red.

Overall, predicate logic mainly includes the following symbols, that is, \&not, $\wedge, \vee$, and $\rightarrow$. Predicate logic is suitable for denoting knowledge properties and situations of things, and the causal relationship between things. Specifically, production rule is superior to predicate logic [4]. 
Production Rules Advantages. (1) Production rule is more accurate. Compared with implication of logic predicate, production rule not only can express inevitable causality, but also can be used to express uncertainty relation as below:

IF P THEN Q (degree of confidence)

Or $\mathrm{P} \rightarrow \mathrm{Q}$ (confidence level)

For example:

IF the patient is the intermediate host

Microbe is Rod-shaped

Gram-negative micro-organisms chromosome plaque presented

THEN the microorganism is Pseudomonas aeruginosa (confidence level 0.6)

(2) Matching criteria is more flexible. When the availability of a piece of knowledge is detected by logic predicate, matching results must be consistent. Unlike the former, production system is to match prerequisite of current knowledge to the known facts, and accuracy of the results can be controlled, as long as the degree of similarity is set in advance within a range. Using a certain matching algorithm can get the knowledge of meeting matching results [5].

\section{Semantic Network Representation}

In addition to the above-discussed and related productions notation, there are other forms of knowledge representation methods, such as semantic network representation, which has powerful representation ability and wide application.

Semantic network is a triples network map with combination of one or more nodes together with the mark and. Each node means to express a fact, event, condition, and other concepts. The pointed of arc is the relationship existed between the facts. Therefore, semantic network reflects the primary and secondary relationship with a directed graph, shown in Figure 3:

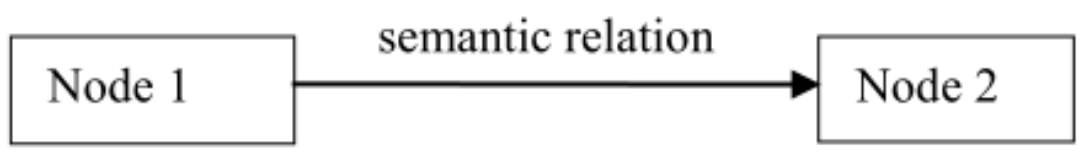

Fig. 3 Primary and Secondary Relationship of Semantic Network

Semantic Relations. Instance relationship: ISA mainly reflects the concepts of abstract and concrete.

Generalization relationship: AKO mainly embodies the concept of super-class and sub-class. Membership: A-Member-of mainly reflects the relationship between individual and collective, shown in Figure 4.

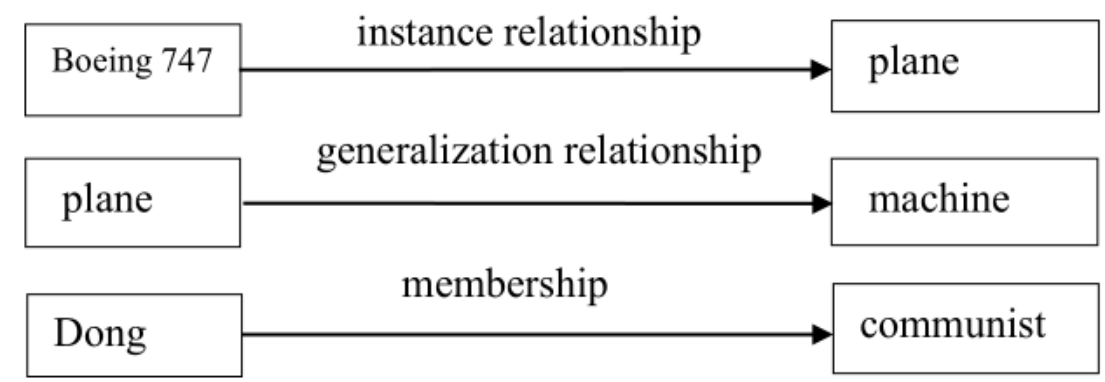

Fig.4 Semantic Relations of Semantic Network

Attribute relationships: refers to the relationship between objects and their properties. Common attributes relations include Have, Can, and Age. Specific semantic network diagram of above relationship is shown in Figure 5.

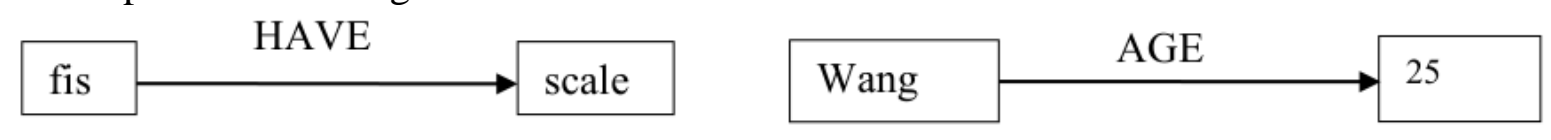

Fig. 5 Properties Relationship of Semantic Network 
Inclusion relation: also called clustering relationships. There is a certain organizational structure between the elements. Common property relations include Part-of, Before, and After. For example, the "heart is part of the body" as shown in Figure 6.

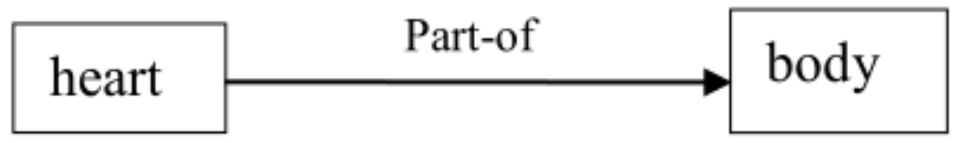

Fig.6 Inclusion Relation

Time relationship: Time relation refers to the occurrence of several events has the differences in time.

Commonly used time relations are Before, After, etc. For example, Qing Dynasty is established after Ming Dynasty, as shown in Figure 7.

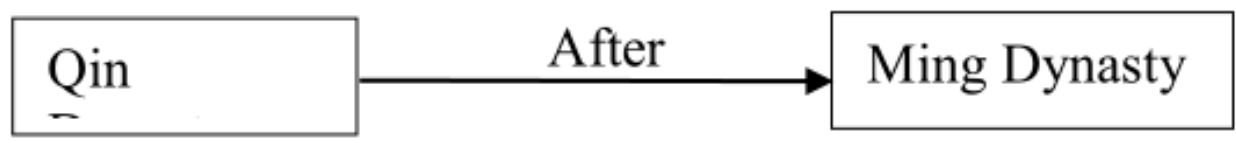

Figure 7 Time Relation of Semantic Networks

Location relationship: Location relationship refers to that there is a clear location relationship between different things. Common location relationship includes Located-under, Located-on, Located-at, Located-inside, and Located-outside For example, computer is on the desk, as shown in Figure 8.

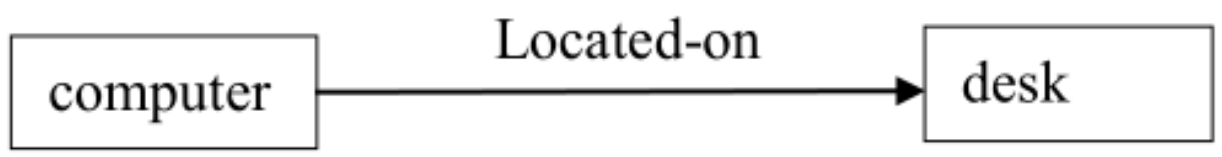

Fig.8 Location Relationship of Semantic Network

Similarity relationship: Several different things are similar or close to some of the properties, such as content, color, material and so on. Commonly used similarity relationship includes Similar-to and Near-to. For example, monkey likes man, shown in Figure 9.

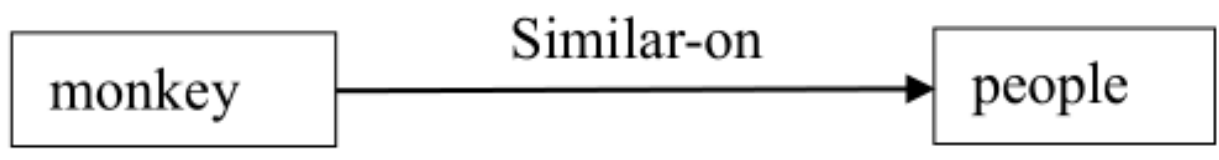

Fig. 9 Similarity Relationship of Semantic Network

\section{Examples of Semantic Representation}

Then, we will explain the four steps of organizing semantic networks through the case as follows:

(1)Object entities of the semantic network include animal, cat, Persian cat and civet. Persian cat and civet cats belong to cats, so they have common attributes, that is, a tail, four legs. A cat belongs to animal, so it has animal attributes.

(2) The relationship between civet cat and cat and the relationship between cat and animal are class relationships, and can also be expressed by generalization relationship.

(3) The same properties in lower nodes and upper nodes should be marked once.

(4) The following Fig.10 of semantic network is generated with some nodes and arcs. 


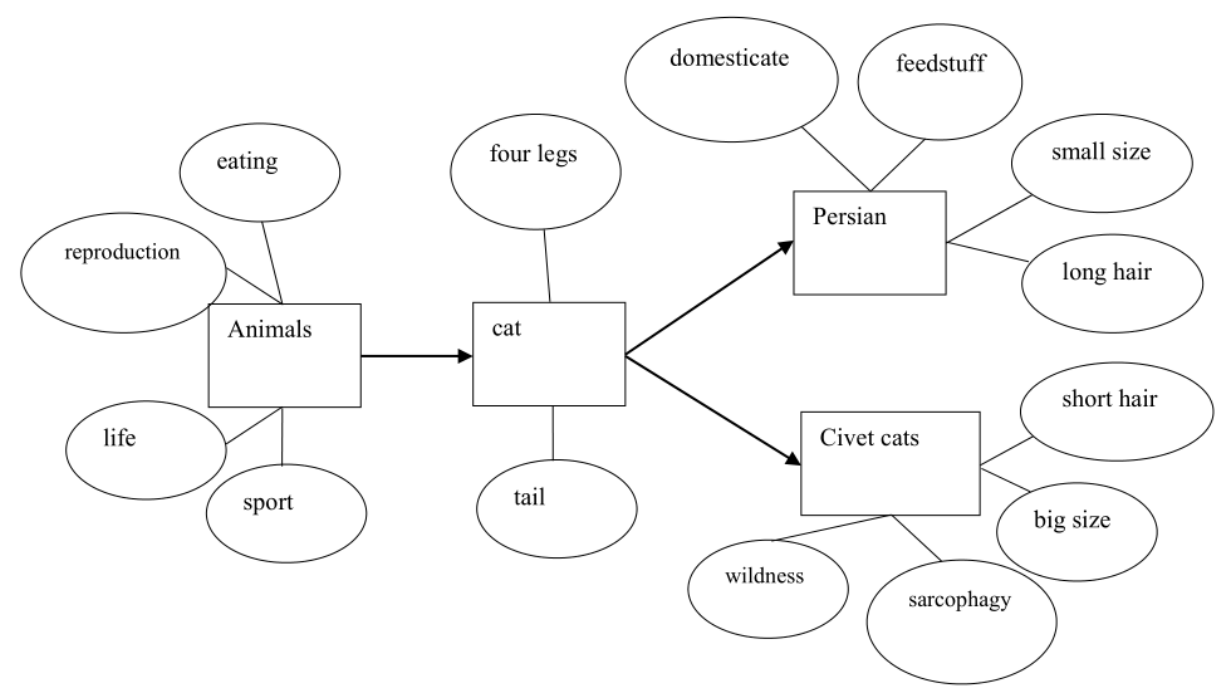

Fig.10 Example of Semantic Network

\section{Conclusion}

This paper analyzed several relationships based on expert system, and compared production rules and predicate logic notation. What's more, advantages of production rules are proposed. Finally, an example was given out according to general procedures of semantic representation. However, there is lots of work to be done in future to improve the process.

\section{Acknowledgement}

The paper is funded by Science and Technology Department of Liaoning Province (No.2012216012) and National Marine Public Welfare Projects (No.201205023-4).

\section{References}

[1]Yang Fuqing, Wang Qianxiang, Mei Hong, et al. Reuse-based software production technology [J]. Science in china (Series F), 2001, 44(1): 8-19.

[2]Raggl A, Slany W A. Reusable iterative optimization software library to solve combinatorial problems with approximate reasoning [J]. International Journal of Approximate Reasoning, 1998, 19(1/2): 161-191.

[3]NATO. Standard for Management of a Reusable software component library [EB/OL]. http://www.uml.org.cn/bzgf/ component/nato_standards_vol_2.pdf. 1991/2009-9-16.

[4]Jean-Guy Schneider, Components, Scripts, and Glue, A concept framework for software composition. PhD dlesis, University ofBern, Deutschland, Oct, 1999

[5]Markus Lumpe, A g-Calculus Based Approach for software Composition, PhD thesis, University ofBern, Deutschland, Jan, 1999 